einen Antrag betreffs Verkauf von Serien der Zeitschrift verhandelt, wozu Wichgraf, Stichel, Kuhnt, Pape und Reineck sprechen. Schlufs $10^{1} / 2$ Uhr.

Aus der entomologischen Welt. Von P. Kuhnt.

Nachrichten aus unserem Leserkreise, besonders vom Ausland, sind jederreit willkommen.

I. Totenlis te.

In Nürnberg verschied am 2. VII. d. J. im Alter von 35 Jahren der Entomologe Max Barthe l. - - Im Juni d. J. starb in Gray (Hte.-Saône) der Hymenopterologe Ernest André, der sich speziell mit Formiciden und Mutilliden beschäftigte. - Am 16. XII. 13 verstarb in South Santa Monica, Kalifornien, der Coleopterologe J. J. Rivers. - Der Professor an der Universität München Dr. Paul A ugust Pauly starb am 9. II. d. J. - Der bekannte Spezialist für Lucaniden und Scarabaeiden Carl Felsche starb am 11. IV. d. J. in Leipzig. - Vor kurzem verstarben noch P. R. Uhler in Baltimore und E. F ̈̈ge in Leipzig. - Am 5. V. d. J. starb in Treptow E. S porreiter. - Am 24. XI. 13 verschied in Reims in 70. Lebensjahre der Coleopterologe LambertAbel Lajoye.

\title{
II. Personalien.
}

Prof. K. M. Heller, Kustos am Zoologischen Museum in Dresden, erhielt den Titel Hofrat. - Dr. E. Hentschel in Hamburg wurde zum Leiter der Hydrobiologischen Abteilung des Hamburger Naturhistorischen Museums ernannt.

\section{Rezensionen und Referate.}

In dieser Rabrik finden im allgemeinen die Besprechungen von Büchern Aufnahmo, welche der Redaktion zar Besprechang in dieser Zeitschrift eingegandt wurden und von welchen der Bibliothek der Gesellschaft ein Exemplar für die Besjrechung überwiesen wird.

A revision of the Ichneumonidae based on the collection in the British Museum (Natural History). Part. III. Tribes Pimplides and Bassides by Cla u de M o r ley, F. Z. S., F. E. S. London, printed by order of the trustees of the British Museum. 1914.

Die vorliegende Arbeit als dritter Teil der Morleyschen Ichneumoniden-Revision enthält die Bearbeitung der Tribus Pimplides und 
Bassides. Die schwierige und bei ihrer weiten Verbreitung auch sehr artenreiche Gattung Xanthopimpla behält sich der Autor für eine besondere Publikation vor. Die zahlreichen, gut durchgeführten Bestimmungstabellen für Gattungen und Arten scheinen recht brauchbar zu sein. - Im Anschlufs an eventuell vorhandene Typen - es handelt sich dabei besonders um Smithsche und Cameronsche Arten - stellt der Verfasser zahlreiche kritische Betrachtungen an, so dafs besonders Arten des letzteren Autors, die ohne Kenntnis der Type nicht zu deuten gewesen wären, klar gestellt sind. Recht dankenswert ist auch die genaue Angabe zahlreicher Fundorte, da summarische Fundortsangaben, ehe nicht die geographische Verbreitung der betreffenden Art genau festgelegt ist, keinen wissenschaftlichen Wert haben. - Aufser den Besprechungen zahlreicher bekannter Arten enthält die vorliegende Arbeit noch die Neubeschreibungen von ca. 50 Spezies, einige Umbenennungen und die Aufstellung der neuen Gattung Friostethus aus "Australasia", die durch einen kragenförmigen Prothorax und aufserordentlich lange Pulvillen ausgezeichnet ist. Eine ganz vortrefflich gelungene Abbildung der Hemipimpla maxima Morl. verdient besonders erwähnt zu werden.

Das ca. 150 Seiten starke Bändchen kann jedem Ichneumonidenforscher nur aufs beste empfohlen werden.

Dr. H. B i s c h of $\mathrm{f}$ - Berlin.

BeiträgezurKenntnis der Land-und Sü fswasserfauna Deutsch-Südwestafrikas. Ergebnisse der Hamburger deutsch-südwestafrikanischen Studienreise 1911. Herausgegeben von W. Michaelsen (Hamburg). Lieferung 1. Hamburg, L. Friederichsen \& Co., 1914. Preis Mk. 12.-.

Die ${ }_{n}$ Hamburger deutsch-südwestafrikanische Studienreise 1911" hatte eine ähnliche Aufgabe wie die in früheren Jahren ausgeführten Hamburger Forschungsreisen. Wie die „Hamburger

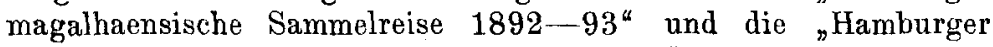
südwest-australische Forschungsreise $1905^{\text {“ sollte sie weiteres }}$ Material für eine tiergeographische Erforschung der 3 in die südlichen Meere hineinragenden Kontinentalspitzen von Süd-Amerika, Süd-Australien und Süd-Afrika herbeischaffen. Durch die Kenntnis der Faunen dieser Gebiete sollten geographische Beziehungen zwischen diesen Erdteilen, wie sie von manchen Forschern vermutet, ja als sicher angesehen werden, aufgeklärt werden. Durch die beiden ersten Reisen waren die tiergeographischen Verhältnisse von Süd-Amerika und Süd-Australien erforscht worden, durch die ${ }_{n}$ Hamburger deutsch-südwestafrikanische Studienreise 1911" sollte 